stimulus, help B cell responses to these host-antigen-worm membrane associations, to produce antibody-mediated death of adult worms? The semi-autoimmune nature of such a response could require for its initiation a heavy infection with its massive antigenc stimulus.

Clearly in infections with protozoan and metazoan parasites, the problems of parasite-host integration and host immune responses are complex. A full understanding of interactions and reactions at the interface between parasite and host is essential if we are to be able to interpret and modify hostparasite relationships to man's advantage.

\title{
Colliding beam fusion
}

from John G. Cordey

THE controlled fusion of the light elements deuterium and tritium has been the main goal of plasma physicists for the past 20 years. The basic problem is one of containing a mixture of deuterium and tritium at a temperature of $10^{\circ}{ }^{\circ} \mathrm{C}$ for a sufficient length of time so that a significant number of fusion reactions take place. At these temperatures the gaseous mixture is a fully ionised plasma which has to be isolated from metallic walls. The main approach to the containment problem has been to use a strong magnetic field to separate the plasma from the walls. Considerable progress has been made using this technique, and toroidal containment devices of the tokamak type are now being designed, which will have parameters similar to those of future fusion reactors (Rebut, Phys. Bull., 26, 264; 1975).

The precise conditions for a reactor to break-even on energetic grounds were first given by Lawson (Proc. Phys. Soc. B70, 6; 1967). For a plasma in thermal equilibrium (that is with Maxwellian distributions of deuterons and tritons) the Lawson criterion requires that the temperature be greater than $5 \mathrm{keV}$, and the product of density and the containment time (the ' $n \tau$ ' product) be greater than $10^{14} \mathrm{~s} \mathrm{~cm}^{-3}$ for break-even.

The colliding beam torus, discussed by Kulsrud and Jassby on page 541 of this issue of Nature, and also by Cordey et al. (Nucl. Fusion, 15, 710; 1975 ) is a toroidal containment device in which the deuterons and tritons, instead of being Maxwellian, are oppositely directed beam-like distributions. These counterstreaming distributions are built up by injecting $60 \mathrm{keV}$ beams of deuterons and tritons into, say, a tokamak device. (This technique of beam stacking has been used for many years in accelerators, at a much lower density of course.)

The mean relative velocity of the two counterstreaming distributions is chosen to be close to the peak of the D.T. fusion cross section at around $120 \mathrm{keV}$. The resulting fusion power output per unit volume of the CBT (colliding beam torus), is thereby much larger than that of a conventional fusion reactor with Maxwellian distributions at say a temperature of $10 \mathrm{keV}$. The conditions for a break-even reactor using the CBT approach are as a consequence far less restrictive. In particular, the $n \tau$ product has only to be greater than $3 \times 10^{12} \mathrm{~s} \mathrm{~cm}^{-3}$ for break-even, rather than the $10^{14}-\mathrm{s}-\mathrm{cm}^{-3}$ demanded by the Lawson criteria for the conventional reactor. Since the energy containment time is thought to scale with the radial dimensions of the device, a small scale experiment demonstrating the efficacy of the CBT approach could be built, whereas a demonstration experiment of the conventional reactor will have to be very large indeed. In fact, the next generation of conventional fusion experiments (such as JET, TFTR) are becoming so large and expensive that in Europe they are having to be built collaboratively.

There are however, it seems to me, several possible difficulties associated with this alternative CBT approach. First, the setting up of the colliding beam configuration requires that the containment time of the thermal plasma is less than the fast ion slowing down time, and that there is no recirculation of the thermal plasma. Although as the authors point out, this first condition is satisfied in present-day tokamaks, the parameters in these machines are very different from those of the CBT. In particular, the mean ion energy is much greater than the electron energy whereas the reverse is the case in tokamaks. In view of the fact that the ion distribution in the CBT is anisotropic, there is a strong possibility that the plasma may suffer from microinstabilities which would reduce the slowing down time of the fast ions. The prevention of recirculation of thermal particles relies on the design of an effective diverter; so far there has been little experimental work on diverters and so this part of the proposal remains somewhat speculative.

Also I have some doubts as to whether the CBT can be developed into an economic reactor in its present form. The quoted values of $Q$, the energy amplification factor, of 4-8 are somewhat smaller than the value of 15 which is said to be necessary for an economic fusion reactor system (Conn et al., Nucl. Fusion, 15, 775; 1975). Even if a pure fusion reactor is not possible however, the high power output per unit volume of the CBT means that it would be eminently suitable as a neutron test facility or with a fissionable blanket as a fission-fusion hybrid reactor.

One further problem that is not mentioned in this particular article, but discussed elsewhere by Jassby (MATT-1145; 1975), is concerned with the penetration of the injected fast neutrals into the plasma. So that the ions leaving the ion sources can move freely across the containing magnetic field, they are neutralised by a gas cell; on entering the plasma they are then re-ionised by the trapped ions. The mean free path of $60 \mathrm{keV}$ deuterium atoms in a plasma of density $n$ is $\left(2 \times 10^{15}\right) / n \mathrm{~cm}$, this places a restriction upon the density or the radius of the device, either of which may affect its reactor potential.

I think to assess whether this novel idea can be developed into a reactor first requires an experimental demonstration of the setting up of the colliding beam configuration. This could probably be done on an existing tokamak with the injection of a few megawatts of, say, $40 \mathrm{keV}$ ions, which is well within the reach of existing neutral beam technology. The scaling of these experiments would then give a clearer indication of the future potential of the CBT as a reactor.

\section{Biological safeguards in genetic engineering}

\section{from David Sherratt}

As well as discussing the types of safeguard needed to eliminate any hazards resulting from genetic manipulation experiments in which genes from potentially any organism can be introduced into and replicated in bacteria, a number of biologists are now attempting to assess experimentally the possible hazards and to develop ways in which they can be minimised (see Nature, 258, 861; 1975). Safeguards fall into two categories-physical containment, which prevents the escape of any possibly hazardous material, and biological containment, which ensures 